\title{
BIOSENSORES PARA CONTROL Y SEGUIMIENTO PATOLOGÍAS REUMATOIDES
}

\author{
Amparo Tirado, Raúl Marín, José V. Martí, Miguel Belmonte, Pedro J. Sanz \\ Departamento de Ingeniería y Ciencia de Computadores, Facultad de Ciencias de la Salud \\ Universitat Jaume I Castellón de la Plana (España) \\ \{al028901,rmarin,vmarti,belmonte,sanzp\}@uji.es
}

\section{Resumen}

El propósito de este artículo es describir el análisis efectuado para obtener un dispositivo que permita mejorar el seguimiento y control de los pacientes con enfermedades inflamatorias como artritis reumatoide $(A R)$, artritis psoriásica (APs) y espondilitis anquilosante (EA). Actualmente este seguimiento se realiza a través de cuestionarios que los propios pacientes deben autocompletar previamente a las visitas con el especialista. Estos formularios aportan información insuficiente, ya que es totalmente subjetiva y depende del estado del paciente en el momento de realizar el cuestionario. Los médicos especialistas consideran que sería una gran ayuda disponer de algún mecanismo no invasivo, que pudiera ser incorporado por los pacientes y estuviera tomando muestras durante el periodo previo a la visita médica.

Para ello se ha realizado un estudio de los biosensores que se están desarrollando actualmente para ver cuál es el que puede proporcionar una información extra para poder realizar un seguimiento más exhaustivo de la evolución de los pacientes.

Queremos que sea un dispositivo de fácil utilización y uso, ya que está orientado a personas mayores, que comienzan a presentar problemas de movilidad.

Palabras clave: Biosensor, Rehabilitación, Reumatoide

\section{INTRODUCCIÓN}

Actualmente los médicos especialistas en enfermedades inflamatorias proporcionan a los pacientes durante sus visitas semanales un cuestionario que éstos deben cumplimentar utilizando para ello sus recuerdos y sensaciones. Unos ejemplos de estos cuestionarios serían.

- MD-HAQ(Multidimensional Health Assessment Questionnaire) ${ }^{1}$.

\footnotetext{
${ }^{1}$ MD-HAQ - http://www.ser.es/wp-content/ uploads/2016/07/MD-HAQ_cuestionario.pdf
}

- EQ-5D (European Quality Of Life) $)^{2}$.

- SF36(36-item Short-Form Health Survey) ${ }^{3}$.

Los cuestionarios se presentan al paciente el día de la consulta o se le entregan para que sean completados el día previo a la consulta con el especialista.

Aquí podemos ver un ejemplo de un cuestionario entregado al paciente.

Versión Española del BASDAI 1

Por favor, marque el número que mejor describa su situación en la ÚLTIMA SEMANA:

1) ¿Cuánta fatiga o cansancio ha tenido usted?

Ninguna $0-1$ Muchísima

2) ¿Cuánto dolor ha tenido usted en cuello, espalda o caderas debido a la espondilitis anquilosante?

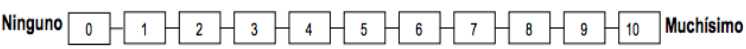

3) ¿Cuánto dolor 0 inflamación ha tenido usted en las otras articulaciones (sin contar cuello, espalda y caderas)?

Ninguno $0-1$ Muchísimo

4) ¿Cuánto malestar ha tenido usted en las partes de su cuerpo que le duelen al tocarlas 0 presionarlas?

Ninguno 0 1 2 (2) 3 Muchisimo

Figura 1: R. Ariza-Ariza, B. Hernández-Cruz y F. Navarro-Sarabia. Rev Esp Reumatol 2004;31(6):372-8 *Diseño: E. Batlle - HGUAlicante

Queremos encontrar un dispositivo lo más completo posible que permita un uso continuado por parte del paciente y ayude al especialista a realizar un seguimiento de la evolución del mismo, para poder ajustar y validar los tratamientos.

En el siguiente artículo podemos ver un estudio similar denominado CAALYX (Complete Ambient Assisted Living eXperiment) [10], dirigido a personas mayores, para su uso en el hogar, que puede medir signos vitales como ECG, pulso,

\footnotetext{
${ }^{2} \mathrm{EQ}-5 \mathrm{D}$ - http://www.ser.es/wp-content/ uploads/2016/07/EQ-5D-pdf.docx

${ }^{3} \mathrm{SF} 36 \quad-\quad \mathrm{https}: / /$ campaign.optum.com/ optum-outcomes.html
} 
presión arterial, detección de movimiento y caída. Es un proyecto de tele-monitorización sobre su localización por medio de brazaletes.

En la Figura 2 se muestra un diagrama del sistema CAALYX

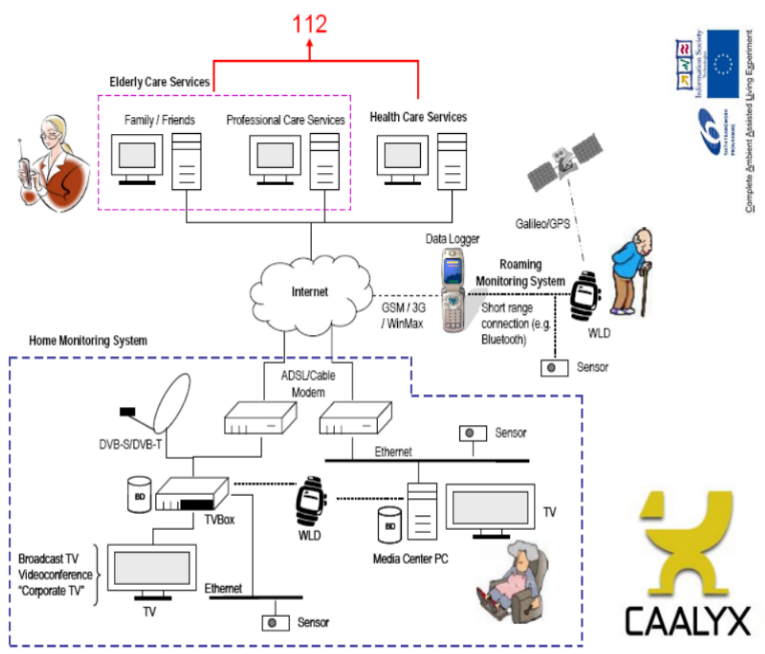

Figura 2: CAALYX system. Diagrama de componentes.

\section{ESTADO DEL ARTE / BIOSENSORES}

\subsection{TIPOS DE SENSORES}

Actualmente existe una gran diversidad de sensores que se están implementando y adaptando para poder ser utilizados como "wearables" , estos sensores tienen multitud de aplicaciones como deportivas, moda, seguridad, industria y aplicaciones médicas.

Nuestro estudio se basa en biosensores desarrollados para aplicaciones médicas y en concreto los biosensores más adecuados para el seguimiento de las enfermedades inflamatorias reumatoides.

Podemos encontrar sensores ubicados sobre la piel, que ya es un nivel donde se puede analizar temperatura, pulso y ritmo respiratorio. Al respecto tenemos el estudio de "Chen, Y., Lu, B., Chen, Y., \& Feng, X. (2015). Breathable and Stretchable Temperature Sensors Inspired by Skin. Scientific Reports, 5, 11505. https://doi.org/10. 1038/srep11505" [1].

Tenemos que priorizar los sensores que cumplan con diversas características, por ejemplo que tengan una autonomía mínima de 48h, deben ser cómodos para el paciente, de poco peso y con la batería integrada. Sería conveniente que pudieran conectarse con dispositivos móviles, de forma que permitan almacenar los datos y enviarlos a su vez a una base de datos que pueda ser consultada por medio de una aplicación parametrizable a medida. Para ello deben ser capaces de incorporar varias tecnologías para la comunicación, algunas de carácter obligatorio, como las conexiones USB y Bluetooth, y otras adicionales, como WiFi o NFC.

Podemos encontrar biosensores que dan soporte a diferentes características, clasificados según tabla adjunta 1 :

Tabla 1: Características biológicas

\begin{tabular}{|l|l|l|}
\hline \multicolumn{3}{|c|}{ Características biológicas } \\
\hline $\begin{array}{l}\text { Temperatura } \\
\text { corporal }\end{array}$ & $\begin{array}{l}\text { Saturación } \\
\text { oxígeno en } \\
\text { sangre }\end{array}$ & Pulso \\
\hline $\begin{array}{l}\text { Presión } \\
\text { sanguínea }\end{array}$ & $\begin{array}{l}\text { Ritmo respi- } \\
\text { ratorio }\end{array}$ & Sudor \\
\hline $\begin{array}{l}\text { EMG Elec- } \\
\text { tromiograma }\end{array}$ & $\begin{array}{l}\text { ECG Elec- } \\
\text { trocardio- } \\
\text { grama }\end{array}$ & $\begin{array}{l}\text { EEG Elec- } \\
\text { troencefalo- } \\
\text { grafía }\end{array}$ \\
\hline \multicolumn{2}{|l|}{ Características físicas } \\
\hline GPS & IPS & Flexibilidad \\
\hline
\end{tabular}

\subsubsection{Características biológicas}

De todas las posibles características a evaluar para el muestreo de sensores nos quedamos con las que mejor de adaptan a los pacientes en estudio; éstas serían :

- Pulso: La función principal del corazón humano es bombear sangre y nutrientes oxigenados al cuerpo y eliminar el dióxido de carbono y otros desechos. La secuencia de ciclos de sangre desoxigenada a través de los pulmones y bombeo de sangre nuevamente oxigenada al cuerpo a través de la aorta se llama secuencia sístole-diástole. La frecuencia cardíaca (HR) o pulso es la frecuencia de la secuencia sístole-diástole, expresada como latidos por minuto (b.p.m.). El HR cambia según la necesidad del cuerpo y es susceptible a la alteración en el estado normal del cuerpo.

El ritmo cardiaco nos ayudar a validar el esfuerzo del paciente y su estudio comparativo nos indica la evolución física del paciente. Como podemos observar en [3], controlar la actividad cardíaca es de gran utilidad. En la Figura 3 podemos ver el esquema utilizado. 


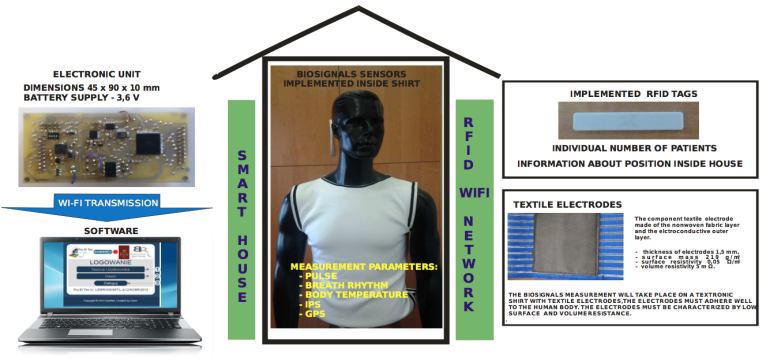

Figura 3: The scheme of the textronics system for protecting of elders. M. Frydrysiak and L. Tesiorowski, "Wearable textronic system for protecting elderly people," 2016 IEEE International Symposium on Medical Measurements and Applications (MeMeA), Benevento, 2016, pp. 1-6.

Existe un trabajo [12] para la monitorización y diagnóstico de diferentes señales como el pulso, la saturación de oxígeno en sangre, que también hace hincapié en la importancia tanto de la medición de datos así como en su posterior interpretación, como se muestra en la Figura 4.

Este dispositivo también cumple una de las premisas de este estudio, que sea "wearable".

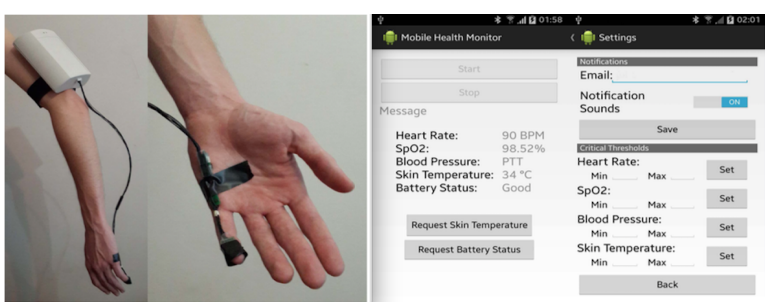

Figura 4: "Body Sensor Network for Mobile Health Monitoring, a Diagnosis and Anticipating System Johan Wannenburg; Reza Malekian IEEE Sensors Journal Year: 2015, Volume: 15, Issue: 12 Pages: 6839 - 6852, DOI: 10.1109/JSEN.2015.2464773 Cited by: Papers (8) IEEE Journals \& Magazines"

- Ritmo respiratorio: El ritmo respiratorio nos ayuda a controlar la fatiga de los pacientes durante su jornada diaria, los que nos indicaría su evolución. En el estudio [7] se muestran los avances en las tecnologías inalámbricas y los diseños de dispositivos más flexibles con una interface mejorada al contacto con la piel.

Los sensores pueden medir de varias maneras al flujo de la respiración, con la expansión y contracción del pecho y el abdomen durante la respiración. Existen numerosos métodos para traducir la expansión y contracción de los pulmones en una señal eléctrica. En la pletismografía de impedancia, los electrodos se colocan sobre el cuerpo y el cambio en la impedancia entre ellos refleja el cambio en el volumen pulmonar durante la inhalación y la espiración. Sin embargo, la mayoría de los sensores de respiración portátiles se expanden físicamente y se contraen junto con los pulmones y emplean algún tipo de deformación o detección de presión para detectar el cambio de volumen. Para este tipo de sensores se utilizan principalmente textiles como podemos ver en la Figura 5 .

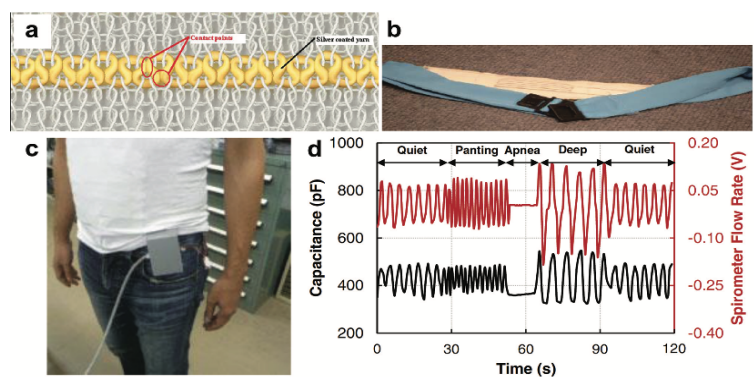

Figura 5: "Monitoring of Vital Signs with Flexible and Wearable Medical Devices Yasser Khan, Aminy E. Ostfeld, Claire M. Lochner, Adrien Pierre, and Ana C. Arias*"
- EMG Electromiograma: Este sensor de músculo mide la actividad muscular por medio del potencial eléctrico generado por las células de la fibra muscular. El sensor amplifica y procesa las complejas señales eléctricas y las convierte en una simple señal análoga que puede ser leída fácilmente por un microcontrolador. En el estudio [6] se indica claramente la utilidad de esta medición. Por este motivo lo consideramos un sensor imprescindible en el estudio y análisis de las enfermedades inflamatorias. Este tipo de sensores han tenido un gran avance en estudios relacionados con exoesqueletos, véase [2], su uso mejora el funcionamiento de los exoesqueletos. La Figura: 6 muestra un esquema de su funcionamiento. 


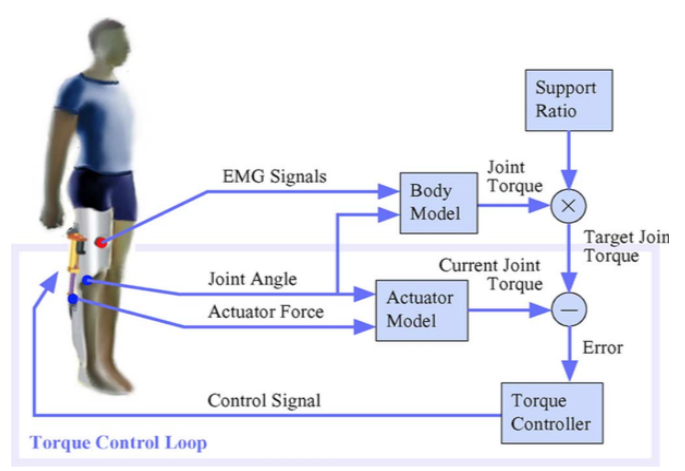

Figura 6: "A Human-Exoskeleton Interface Utilizing Electromyography Christian Fleischer and Günter Hommel"

\subsubsection{Características físicas}

- GPS: Para poder realizar un seguimiento completo del paciente, debemos controlar su movilidad, para poder evaluar sus funciones físicas y así poder comparar los datos a lo largo del tratamiento. Para ello nada mejor que un sensor de movimiento, como por ejemplo unidades de medición inercial (IMU) y el uso de la acelerometría para calcular el gasto energético [5]. Este tipo de sensores tienen gran presencia comercial, por ejemplo https://www.invensense.com/ products/motion-tracking/9-axis/

- IPS: Un sistema de posicionamiento interior nos puede ayudar a controlar la movilidad del paciente dentro de su vivienda o en centros hospitalarios tal y como se indica en [3]. Con un sistema de balizas se pueden emplear técnicas de localización como la mostrada en [3].

- Protocolo de comunicación: La elección del protocolo de comunicación es un paso importante. Lo basaremos en el coste, la facilidad de configuración y de incorporar nuevos sensores. En el estudio [9] vemos una tabla comparativa.

- Flexibilidad: Es importante tener en cuenta la ergonomía del dispositivo, para poder evitar entre otras características diseños demasiado voluminosos o rígidos. Así mismo, es interesante intentar dotarlos de baterías de larga duración o que permitan la generación de energía. En la Figura 7 se muestra una imagen del prototipo propuesto por [11].

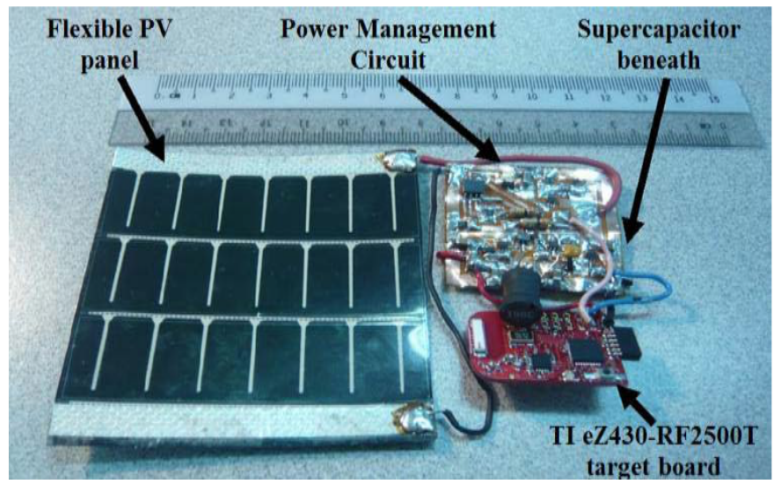

Figura 7: "Final integrated FEH system prototype. Autonomous Wearable Sensor Nodes with Flexible Energy Harvesting."

Existen numerosos estudios que tratan la durabilidad de las baterías e incluso dispositivos "SoC" capaces de obtener su propia energía [13]. El chip realiza la extracción de la frecuencia cardíaca del ECG y la detección de la fibrilación auricular mientras que solo consume $19 \mathrm{uW}$, funcionando solamente en la energía obtenida. En la Figura 8 se muestra el diseño del chip.

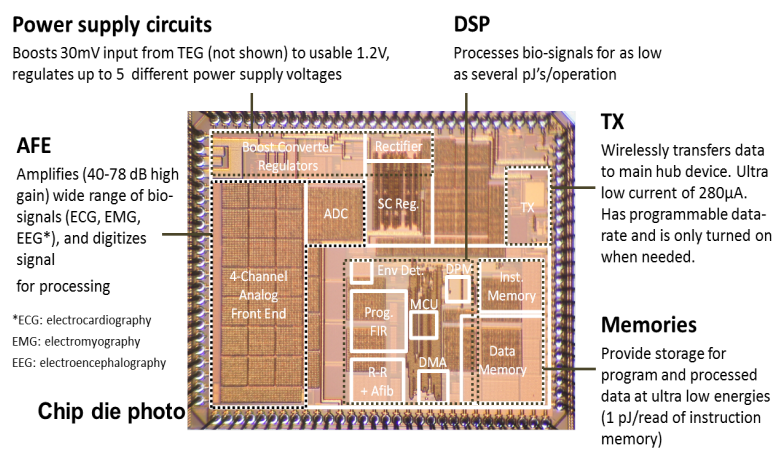

Figura 8: "A Batteryless 19 W MICS/ISM-Band Energy Harvesting Body Sensor Node SoC for ExG Applications"

\section{PRODUCTOS COMERCIALES}

\subsection{Simband modular health band}

Plataforma de código abierto, https: //www.simband.io/documentation/ simband-documentation/, mostrado en las Figuras 9, 10 , 11 y 12 


\section{Simband}

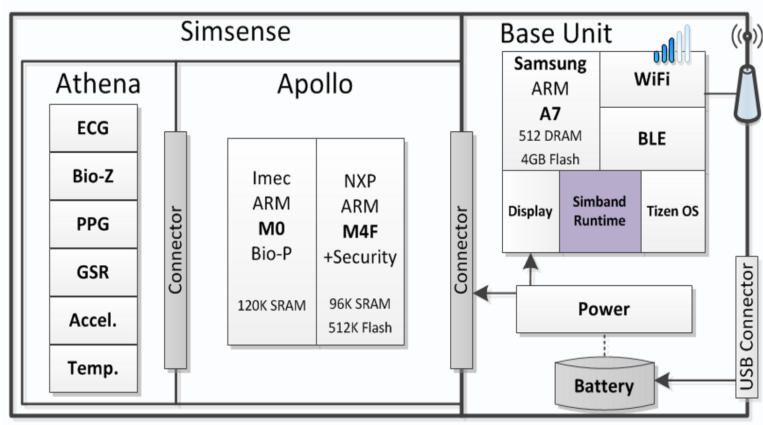

Figura 9: "Simband- Diagrama"

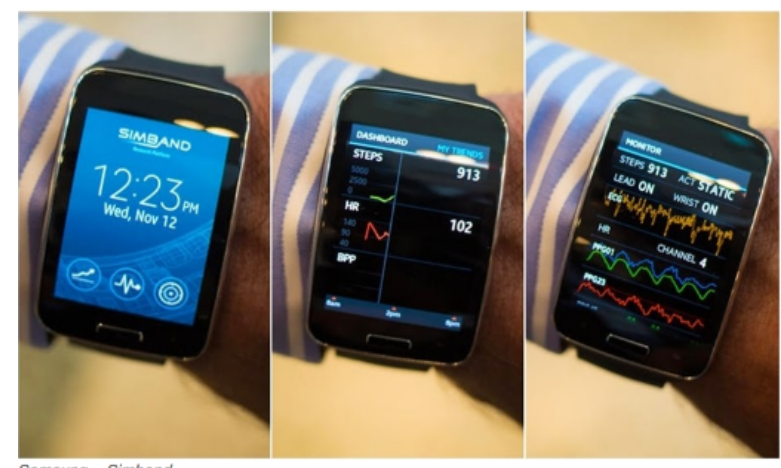

Figura 10: "Simband"
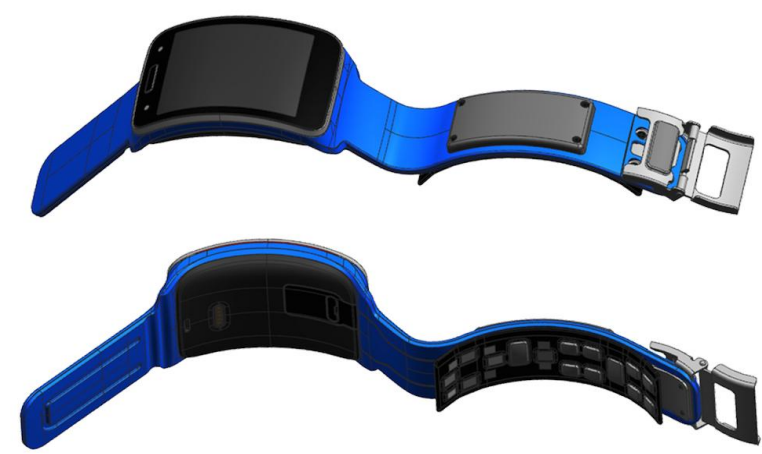

Figura 11: "Simband - Diseño"

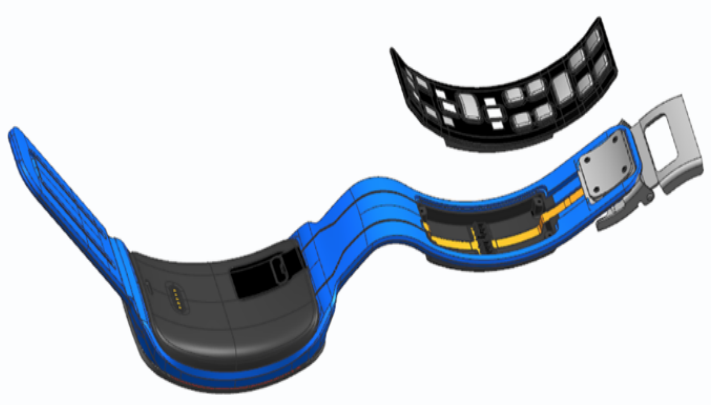

Figura 12: "Simband - Sensores"

\subsection{Empatica E4 Wristband}

Este brazalete [4], mostrado en la Figura 10, recoge continuamente el ritmo cardíaco, la actividad electrodérmica, la temperatura y la actividad física mediante tres acelerómetros. Con las aplicaciones desarrolladas para este sensor, se puede monitorizar la salud del paciente mientras está en su casa, o mediante una aplicación móvil por los familiares.

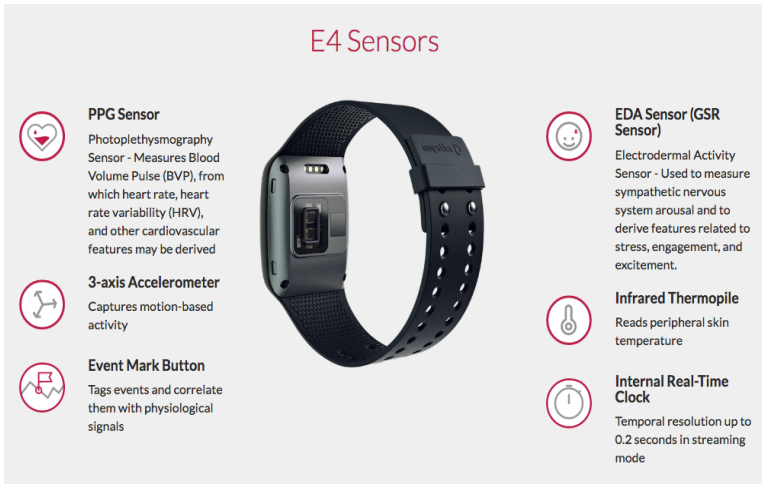

Figura 13: "Empatica E4 Wristband"

Las características completas las podemos encontrar en la web corporativa https://www. empatica.com/e4-wristband

\section{3 e-Z430-Chronos}

Dispositivo de Texas Instruments http://www. ti.com/solution/wireless_patient_monitor, consistente en un reloj de aspecto normal, mostrado en la Figura 14, dotado de acelerómetro y sensor de temperatura corporal, al que pueden ser adaptados sensores inalámbricos permitiendo obtener ECG, niveles de oxígeno y otros parámetros del paciente. 

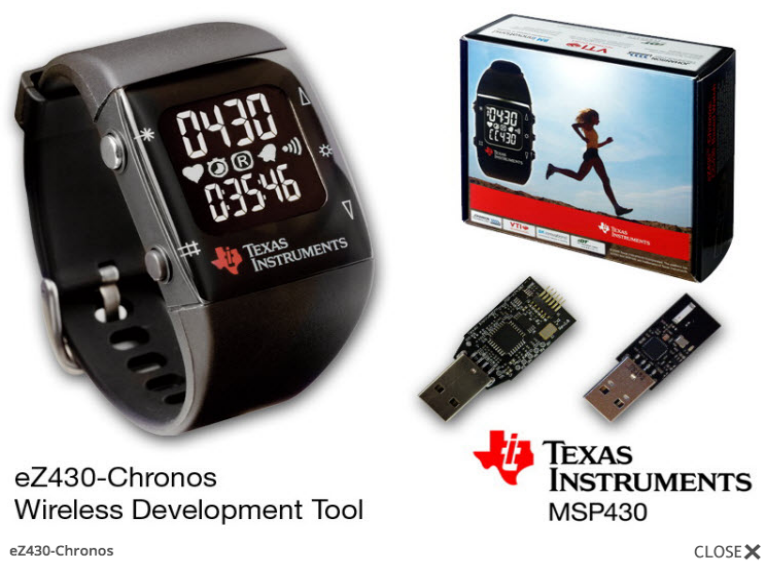

Figura 14: "eZ430-Chronos

\section{COMPARATIVA}

Todos los productos comerciales que hemos estudiado poseen de un API que nos permite crear nuestras propias aplicaciones para el tratamiento de los datos obtenidos. En la Tabla 2, podemos ver que son pocos los productos actualmente comercializados que incorporen varios sensores en un mismo dispositivo.

Tabla 2: Comparativa Productos Comerciales

\begin{tabular}{|c|l|l|l|l|l|r|}
\cline { 2 - 7 } \multicolumn{1}{c|}{} & \multicolumn{5}{c|}{ Características } \\
\hline Productos & GSR & ECG & Acc & Temp & Pulso & Coste \\
\hline Simband & $\mathrm{x}$ & $\mathrm{x}$ & $\mathrm{x}$ & $\mathrm{x}$ & & - \\
\hline Empatica & $\mathrm{x}$ & & $\mathrm{x}$ & $\mathrm{x}$ & $\mathrm{x}$ & $1.521 \$$ \\
\hline Chronos & & $\mathrm{x}$ & & & & $58 \$$ \\
\hline
\end{tabular}

\section{CONCLUSION}

Necesitamos un sensor que tenga buena aceptación por parte de los pacientes [8], que suponga para ellos un aliciente y no les cause rechazo. Debe ser capaz de monitorizar su actividad diaria, almacenarla y proveer de un mecanismo sencillo y configurable para la obtención de los resultados.

Hemos de tener en cuenta los parámetros que es capaz de medir, la conectividad del sistema, la durabilidad de su batería, la interfaz de la que dispone, así como los materiales, para que pueda ser flexible y duradero.

Actualmente no existen en el mercado dispositivos que se adapten completamente a nuestros objetivos. Hemos podido observar que se están realizando numerosos avances técnicos en cuanto a la mejora de los sensores, su flexibilidad, que los hace mas cómodos y su durabilidad, con baterías que se autorrecargan.
Si pensamos en productos que existan actualmente en el mercado son pocos los que se dedican al área de salud, ya que la gran mayoría avanza hacia área deportivas. Los que se están desarrollando son dispositivos que se integran en un sistema para motorizar pacientes en entorno clínicos, son complejos de manejar y no pueden ser utilizados por el propio paciente en el hogar, un ejemplo sería el Drager Infinity M540 ${ }^{4}$.

Muy pocos están dirigidos hacía personas mayores, teniendo en cuenta el envejecimiento de la población mundial y el aumento de las esperanza de vida, pensamos que hemos de hacer lo posible por mejorar el seguimiento y control de este sector de la población para ayudar a la detección precoz de dolencias propias de la edad, así como poder validar mejor el funcionamiento de los distintos tratamientos prescritos a los pacientes.

\section{Agradecimientos}

El presente trabajo está soportado por la Universidad Jaume I, en colaboración con la Sección de Reumatología del Hospital General de Castellón, a través del Departamento de Ingeniería y Ciencia de los Computadores, grupo de investigación en robótica IRSLab (www.irs.uji.es). Por el gran interés del uso de biosensores para monitorizar el estado del usuario en el momento de intervenciones robóticas de rescate, el presente trabajo está parcialmente soportado por los siguientes proyectos, MINECO DPI2014-57746-C31-R (MERMANIP Project), Generalitat Valenciana GVA-PROMETEO/2016/066, y Universidad Jaume I de Castellón (P1-1B2015-68)

\section{Referencias}

[1] Chen, Y., Lu, B., Chen, Y., And Feng, X. Breathable and Stretchable Temperature Sensors Inspired by Skin. Scientific Reports 5, 1 (sep 2015), 11505.

[2] Fleischer, C., AND Hommel, G. A human-exoskeleton interface utilizing electromyography. IEEE Transactions on Robotics 24, 4 (Aug 2008), 872-882.

[3] Frydrysiak, M., And Tesiorowski, L. Wearable textronic system for protecting elderly people. In 2016 IEEE International Symposium on Medical Measurements and Applications (MeMeA) (May 2016), pp. 1-6.

[4] Garbarino, M., Lai, M., Bender, D., PiCARD, R., And Tognetti, S. Empatica E3

\footnotetext{
${ }^{4}$ Drager - https://www.draeger.com/en-us_ us/Hospital/Products/Patient-Monitoring/ Patient-Monitors/Inf inity-M540-monitor
} 
- A wearable wireless multi-sensor device for real-time computerized biofeedback and data acquisition. 39-42.

[5] Grimm, B., And Bolink, S. Evaluating physical function and activity in the elderly patient using wearable motion sensors. EFORT Open Reviews 1, 5 (2016), 112-120.

[6] Gutierrez-Gutierez, G., Barbosa Lopez, C., Navacerrada, F., AND Miralles Martinez, A. Utilidad del electromiograma en el diagnostico de las miopatias inflamatorias. Reumatologia Clinica 8, 4 (2012), 195-200.

[7] Khan, Y., Ostfeld, A. E., Lochner, C. M., Pierre, A., And Arias, A. C. Monitoring of vital signs with flexible and wearable medical devices. Advanced Materials 28, 22 (2016), 4373-4395.

[8] Mercer, K., Giangregorio, L., SchneiDer, E., Chilana, P., Li, M., AND Grindrod, K. Acceptance of Commercially Available Wearable Activity Trackers Among Adults Aged Over 50 and With Chronic Illness: A Mixed-Methods Evaluation. JMIR mHealth and uHealth 4, 1 (jan 2016), e7.

[9] Mukhopadhyay, S. C. Wearable sensors for human activity monitoring: A review, 2015.

[10] Rocha, A., Martins, A., Freire, J. C., Kamel Boulos, M. N., Vicente, M. E., Feld, R., VAN DE VEn, P., Nelson, J., Bourke, A., ??Laighin, G., SDOgati, C., Jobes, A., Narvaiza, L., And RoDr??GUEz-MOLINERo, A. Innovations in health care services: The CAALYX system. International Journal of Medical Informatics 82, 11 (2013).

[11] Toh, W. Y., Tan, Y. K., КоH, W. S., AND SIEK, L. Autonomous wearable sensor nodes with flexible energy harvesting. IEEE Sensors Journal 14, 7 (2014), 2299-2306.

[12] Wannenburg, J., And Malekian, R. Body sensor network for mobile health monitoring, a diagnosis and anticipating system. IEEE Sensors Journal 15, 12 (Dec 2015), 6839-6852.

[13] Zhang, Y., Zhang, F., Shakhsheer, Y., Silver, J. D., Klinefelter, A., NAgaraju, M., Boley, J., Pandey, J., Shrivastava, A., Carlson, E. J., Wood, A., Calhoun, B. H., and Otis, B. P. A batteryless 19 uw mics/ism-band energy harvesting body sensor node soc for exg applications.
Journal of Solid State Circuits 48 (01/2013 2013), 199-213. 